\title{
Reconfigurable Intelligent Surface-Assisted Bluetooth Low Energy Link in Metal Enclosure
}

\author{
Mir Lodro ${ }^{1 *}$, Gabriele Gradoni ${ }^{1,2}$, Jean-Baptiste Gros ${ }^{3,4}$, Steve Greedy ${ }^{1}$ and \\ Geoffroy Lerosey ${ }^{3}$
}

${ }^{1}$ Radio Frequency Laboratory, George Green Institute for Electromagnetic Research-GGIEMR, University of Nottingham, Nottingham, United Kingdom, ${ }^{2}$ School of Mathematical Sciences, University of Nottingham, Nottingham, United Kingdom, ${ }^{3}$ CNRS, The Institut Langevin, ESPCI Paris, PSL University, Paris, France, ${ }^{4}$ Greenerwave, Paris, France

OPEN ACCESS

Edited by:

Ertugrul Basar,

Koç University, Turkey

Reviewed by:

Ibrahim Yildirim,

Istanbul Technical University, Turkey

Wankai Tang,

Southeast University, China

*Correspondence:

Mir Lodro

(mir.lodro,mir.lodro1)

@nottingham.ac.uk

Specialty section:

This article was submitted to

Wireless Communications,

a section of the journal

Frontiers in Communications and

Networks

Received: 30 June 2021

Accepted: 16 August 2021

Published: 26 October 2021

Citation:

Lodro M, Gradoni G, Gros J-B,

Greedy S and Lerosey G (2021)

Reconfigurable Intelligent SurfaceAssisted Bluetooth Low Energy Link in

Metal Enclosure.

Front. Comms. Net 2:733637.

doi: 10.3389/frcmn.2021.733637
Reconfigurable intelligent surface (RIS) technology is at the forefront for its transformative role in future wireless communication systems such as wireless local area networks (WLAN), sixth-generation (6G) communication, and internet-of-things (IoT). This paper presents RIS-assisted Bluetooth low energy (BLE) communication links in neighbor discovery mode. We optimized the packet error rate (PER) performance of the BLE communication link in a highly reflecting metal enclosure environment. We used one RIS for the PER optimization of four BLE physical (PHY) modes. Then, we used two RISs simultaneously in a distributed and centralized manner to further optimize the PER of all BLE PHY modes. We found PER optimization using two RISs is better than the PER optimization using one RIS. Additionally, PER optimization using a centralized arrangement of RISs outperformed PER optimization using distributed arrangement. We found the coded BLE modes i.e., LE500K and LE125K show lower PER than the uncoded counterpart i.e., LE1M and LE2M. This is because uncoded BLE PHY modes have higher data rates than the coded BLE PHY modes. Because of additional channel power gains introduced by RIS-based passive beamforming, the PER of coded and uncoded BLE PHY modes is further reduced.

Keywords: bluetooth low energy, reconfigurable intelligence surface, loT-internet of things, metal enclosure, plutoSDR

\section{INTRODUCTION}

Reconfigurable intelligent surface (RIS) is a revolutionizing technology which can configure wireless propagation environment by using a large number of low-cost passive reflecting elements where each passive element can induce amplitude and phase shifts thus beamforming and nulling the signal in an area of the interest (Kaina et al., 2014b; Basar et al., 2019; Tang et al., 2020a). RIS can be used to coat planar or conformal objects in the environment that constitute a radio channel such as walls, ceilings, building facades, furniture. Therefore, RIS can address the core problems of energy-efficiency, spectral-efficiency, co-channel interference, PHY layer security (Cui et al., 2019; Xu et al., 2021). Hence, RIS shall be central to 6G wireless communication Calvanese Strinati et al. (2021). Received power in RIS-assisted communication system is proportional to the number of elements used in the RIS, reflection coefficient and reflection-efficiency of the elements. Therefore, a large RIS with higher number of passive reflecting elements can optimize BLE link effectively. RIS do not use amplifiers, analog to digital converter/ digital to analog converter (ADC/DAC) and active RF chains therefore it 
consumes significantly less energy than the contemporary massive MIMO, active amplify and forward relaying technique Björnson et al. (2019). However, there are works which suggest usage of active sensor components in addition to passive elements for both pilot-based channel estimation and passive beamforming. A detailed assessment and performance analysis of reconfigurable intelligent surface and relaying in terms of diversity gain and end-to-end SNR is given in Boulogeorgos and Alexiou (2020). Authors in $\mathrm{Hu}$ et al. (2020) have developed experimental framework for RF sensing of RISassisted posture recognition. Authors in Dai et al. (2020), Tang et al. (2020b) have demonstrated RIS-based experimental prototypes for demonstration of wireless communication system. Authors in Dai et al. (2020) developed 256 element RIS prototype and demonstrated wireless communication systems with antenna gains of $21.7 \mathrm{dBi}$ and $19.1 \mathrm{dBi}$ at $\mathrm{RF}$ frequency of 2.3 and $28.5 \mathrm{GHz}$. A framework for joint trajectory and passive beamforming using RIS unmanned aerial vehicle (UAV) communication has been presented in Li et al. (2020). RIS is a cost-effective, energy-efficient and spectral-efficient solution for sixth-generation wireless communication as it will solve key technical issue of blockage at mmWave band and $\mathrm{THz}$ band where enormous bandwidth can be exploited to meet the user bandwidth requirements (Wu and Zhang, 2019; Yuan et al., 2021). Authors in Tan et al. (2018) have demonstrated RISassisted indoor link for IEEE $802.11 \mathrm{ad}$ at $60 \mathrm{GHz}$. RIS application at mmWave band can be used to overcome the sparsity of mmWave channels. Energy-efficiency of RIS has been studied by authors in Huang et al. (2019). RIS can also be useful for secondary user link optimization in cognitive radio and alleviating interference to primary user link Guan et al. (2020). Authors have presented sum-rate maximization of secondary user link subject to minimizing interference to primary user.
Authors in Hou et al. (2019) have studied the issue of co-located multi-RIS deployment. The applications of RISs are diverse. We take the opportunity to propose the usage of RIS for low energy Bluetooth. BLE is a key short-range data transmission technology in internet of things along with Zigbee and narrowband internet of things (NB-IoT) that is used for multimedia file sharing, home automation, and other internet-of-things applications such as medical internet of things, smart homes, indoor positioning, and localization (Montoliu et al., 2020; Spachos and Plataniotis, 2020). We believe the usage of RIS in BLE framework can increase the signal quality, PER performance and also increase the overall lifetime of already energy-constraint IoT devices. In this work, we arranged master and slave BLE nodes in NLOS in a reverberant metal enclosure, an environment that has been tackled by other investigators in wireless communication (Gagliardi et al., 2015; Micheli et al., 2016; Barazzetta et al., 2017; Primiani et al., 2020; Micheli et al., 2021), as shown in Figure 1. We have optimized the PER performance using RIS of all the BLE PHY modes. We have divided our manuscript into four sections. Section $\mathbf{1}$ is introduction and motivation of the usage of RIS technology in BLE communication. Section 2 explains BLE baseband transmitter and receiver for BLE PER measurement as well as the measurement setup. Section 3 is measurement result about PER optimization using RIS. Finally, the conclusion is presented in section 4 .

\section{BLE BASEBAND TX/RX MODEL AND MEASUREMENT SETUP}

\subsection{BLE Baseband Transmitter and Receiver}

Baseband transmitter generates link layer PDUs and generates IQ waveforms which are passed to PlutoSDR for over-the-air

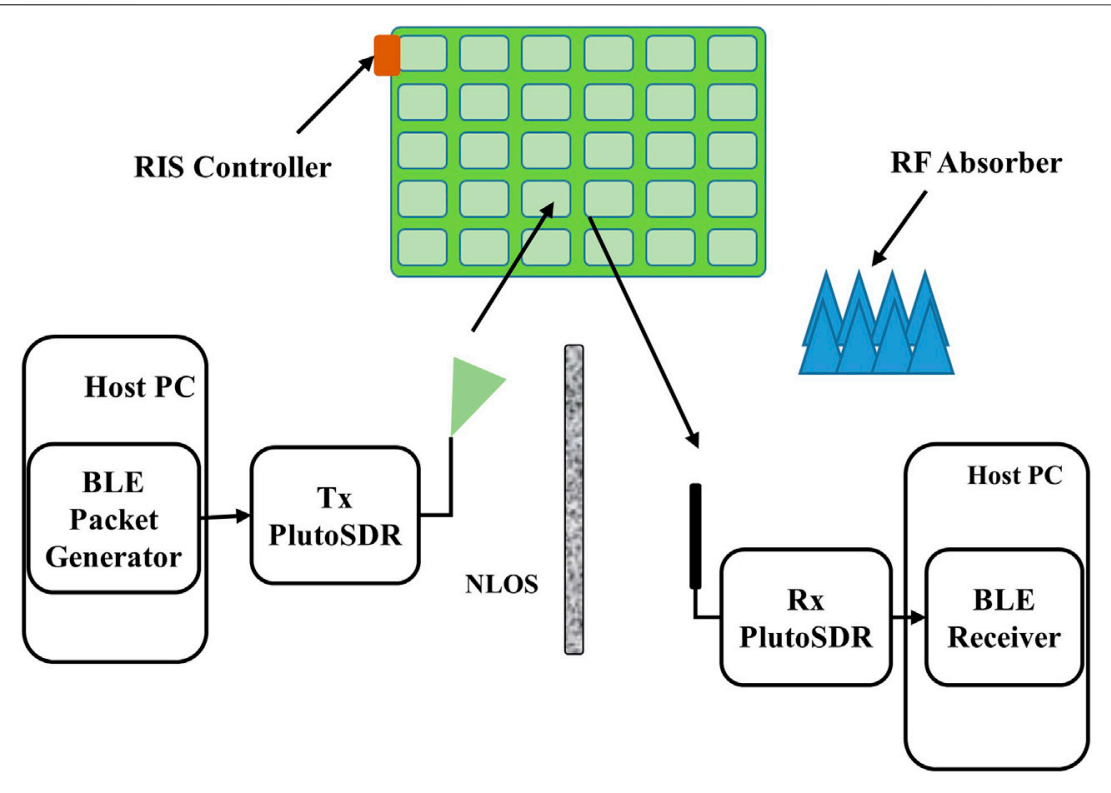

FIGURE 1 | RIS-assisted BLE PER measurement sketch. 
transmission. Link layer PDUs are modelled as advertising channel PDUs or data channel PDUs. BLE receiver performs RF impairment compensation such as RF nonlinearity effect, carrier frequency offset, carrier phase offset, and symbol timing offset. Standard-compliant BLE receivers consist of automatic gain control (AGC) stage, DC compensation stage, matched filtering, carrier frequency offset (CFO) estimation, timing synchronization stage, demodulation, de-whitening, and CRC stage. After the initial receiver operations demodulation of Gaussian minimum shift keying (GMSK) symbols is accomplished and a CRC check is performed. The AGC component in the digital receiver is vital for the correct recovery of information and for end-to-end PER performance measurement. The AGC is the first stage where a weak signal is received and stabilized for further downstream processing. The AGC can be configured in slow-attack mode or fast-attack mode to track the received signal. Previously, we have recorded our experimental experience with AGC in the metal enclosure Lodro et al. (2020). The AGC helps for coarse and fine frequency correction in the baseband receiver. AGC is configured with maximum power gain of $20 \mathrm{~dB}$ and desired power of $2 \mathrm{~W}$. After the AGC stage, DC removal in the received waveform is performed using a notch filter. The existence of the DC component is a common problem in radio receivers, and it is particularly common in direct-conversion receivers such as software-defined-radios. The next two stages are frequency offset correction and matched filtering. Frequency offset estimation and correction must be performed before received samples are processed by a matched filter. The matched filter increases the signal-to-noise (SNR) at the receiver i.e., it limits the amount of noise that is passed down to subsequent stages Litwin (2001) in the receiver. Coarse frequency compensation uses an FFT-based or correlation-based algorithm to measure frequency offset. Fine frequency and demodulation of GMSK packets are performed. Frame timing synchronization is performed using preamble detection which measures detection metric and the threshold. After these critical design stages, data decoding and demapping are performed. Long series of zeros and ones such as 000000 or 1111111 may occur in the data bitstream. Data whitening is used to avoid such a long sequence of ones and zeros. Data de-whitening and CRC are performed to measure the BLE PER. For the packet error measurement, access address is checked first and then CRC is checked, If the access address or $\mathrm{CRC}$ is incorrect then the packet shall be rejected. The received BLE packets are considered only valid when both access address and CRC check is correct. Figure 2 shows flowchart of the BLE link PER measurement process. First BLE PHY mode specific packetized baseband waveforms are generated which are then digital upconverted for over-the-air transmission. After the NLOS channel is tuned using RIS elements, the BLE packet capture is performed using $\mathrm{Rx}$ PlutoSDR. The captured packets are further processed using baseband BLE receiver at host PC. BLE operates using uncoded and coded PHY modes with minimum and maximum data-rates of $125 \mathrm{Kbps}$ for coded and 2 Mbps for uncoded respectively. Packet format for uncoded and coded BLE PHY modes consists of 32-bit access address, 16-bit header length, 24-bit cyclic redundancy check (CRC),

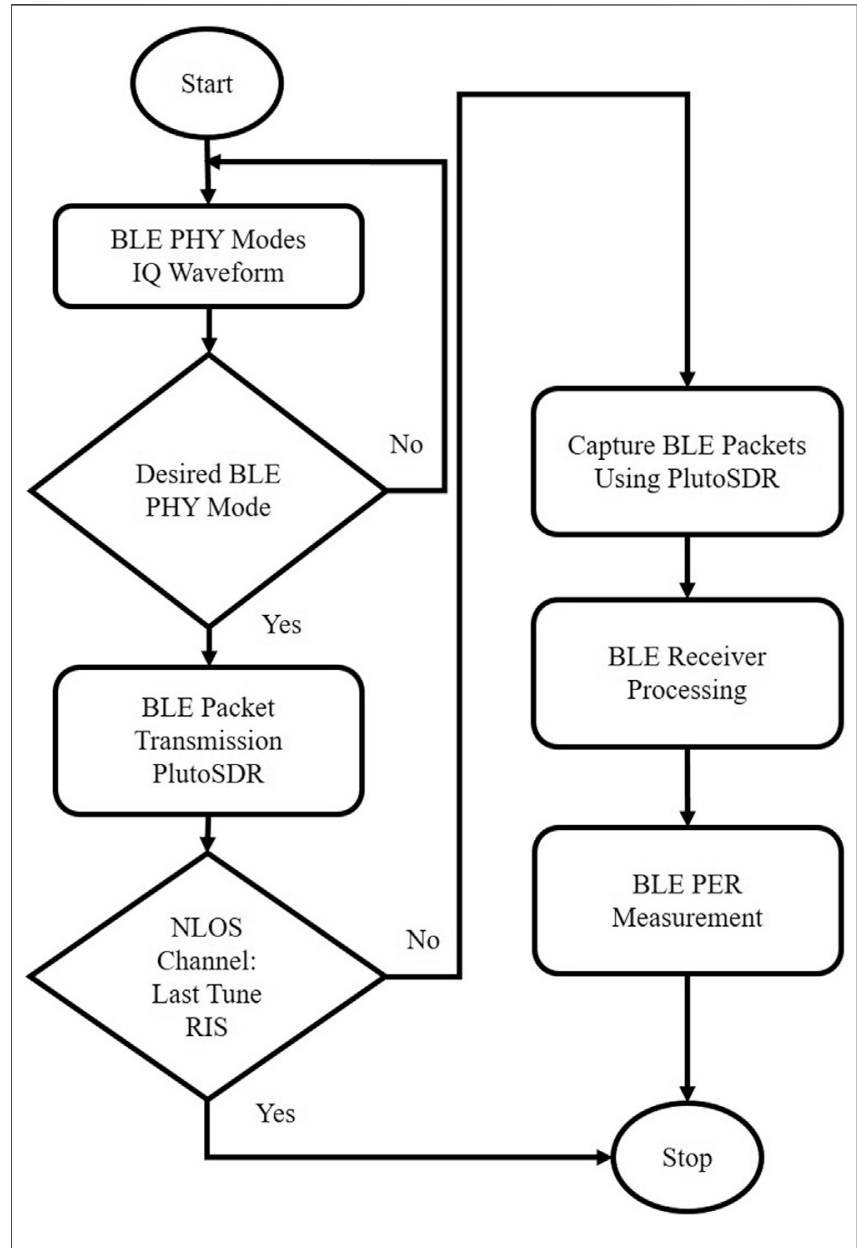

FIGURE 2 | RIS-assisted BLE PER measurement flowchart.

and 16-bit to 2056-bit protocol data unit (PDU) with additional term 1 and term2 fields for coded BLE PHY modes. Preamble field for uncoded and coded BLE PHY mode is 8/16-bit and 80-bit, respectively. We presented PER measurement of BLE in presence of WLAN interference in reverberation chamber but without reconfigurable intelligent surface Lodro et al. (2021).

\subsection{Measurement Setup}

We created BLE communication link in neighbor discovery mode in the metal enclosure. The metal enclosure used has dimensions of $h \times \ell \times w$ of $1 \times 0.8 \times 1 \mathrm{~m}^{3}$. We used two PlutoSDRs from Analog Devices Inc. (ADI) for BLE communication link in metal enclosure. PlutoSDR is a low-cost full-duplex transceiver with ADI 9363 integrated RFIC with operating frequency range from $325 \mathrm{MHz}$ to $3.8 \mathrm{GHz}$ and its RF frequency range is software upgradeable upto $6 \mathrm{GHz}$. The transmitter $(\mathrm{Tx})$ and receiver $(\mathrm{Rx})$ PlutoSDRs are connected with host PC for exchanging transmitted and received complex signal samples. We also connected RISs via USB-serial port with the same host PC. Each RIS plate consists of eight rows and ten columns of passive elements. Therefore, the RIS can introduce discrete 


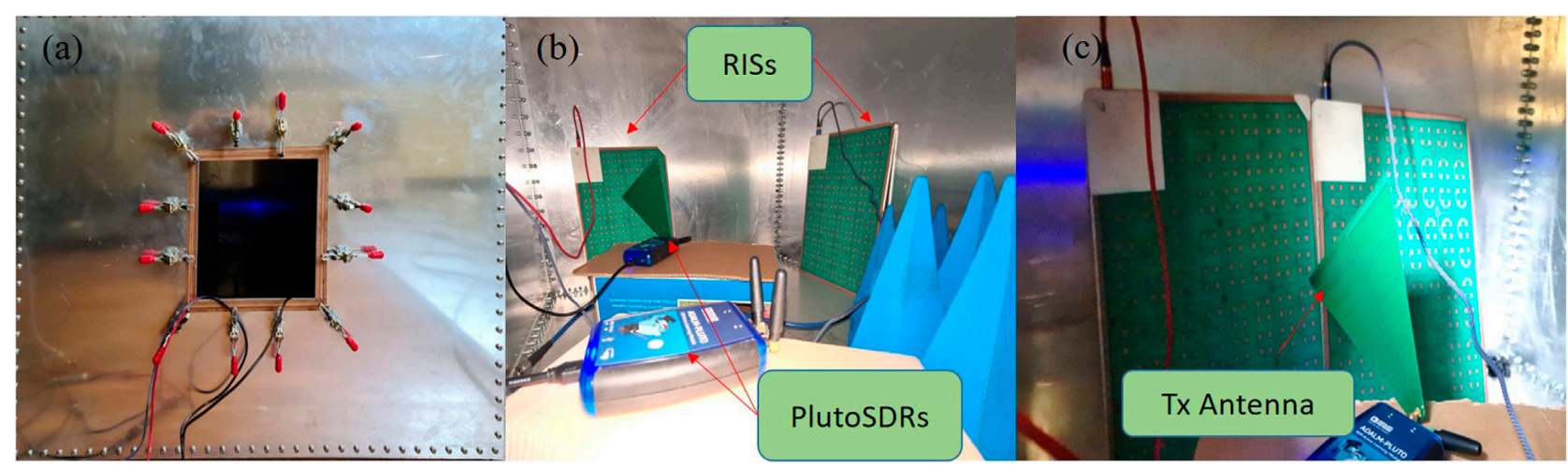

FIGURE 3 | Measurement setup (A) metal enclosure (B) distributed RISs (C) centralized or co-located RISs.

phase shifts to incident signal. We also connected RIS manufactured and patented by GREENERWAVE (https:/greenerwave.com/) to the same host PC through USB serial port. The same USB port is used to simultaneously power the integrated control board of the RIS and send commands that impose RIS configuration by controlling the bias voltage of a set of PIN diodes through a set of shift registers. Each surfaces consists of 76 phase-binary pixels, which are based on hybridizing two resonances Kaina et al. (2014a). Each pixel can be configured electronically to control independently, via the states of two PIN diodes, both components of the reflected electric field tangential to the pixel plane by imposing a 0 or $\pi$ phase, thus leading to a total number of 152 effective pixels per RIS. The transmitter and receiver BLE baseband models run in separate MATLAB sessions. We considered RIS-assisted non-LOS (NLOS) single-input single-output (SISO) BLE communication link by using directional Tx antenna. The direction of peak radiation of transmitting antenna is pointed towards RIS. This means the multipath contribution at the receiver is largely from the RIS. There is line-of-sight (LOS) between transmitter and the RIS for leveraging maximum benefits from RIS beamforming. RIS can be deployed closer to transmitter or closer to user to fully exploit RIS passive beamforming. In our work, transmitter is located closer to RIS and there is no direct link between transmitter (Master) and receiver (Slave). Hence, PER measurement takes place using cascaded channel i.e. Master-RIS-Slave link in neighbour discovery (NDP) mode. BLE PHY mode specific waveforms are generated using MATLAB-based BLE baseband transmitter. Complex waveform samples are transferred to Tx PlutoSDR for over-the-air transmission in metal enclosure. The metal enclosure is perfectly reflecting environment and it supports rich multipath scattering. The measurements are conducted in stationary environment i.e. there is no active modestirrer inside metal enclosure except two PlutoSDRs, two RISs and ten pieces of standard RF absorber cones. We conducted BLE PER measurements using one RIS with Tx antenna pointing towards RIS as shown in Figure 3. Then we conducted PER optimization of BLE modes using two RISs in distributed and centralized arrangement. In distributed arrangement, the RIS 1 was located closer to Tx and the RIS 2 was located at some distance from RIS 1. Similarly, in centralized arrangement RIS 1 and RIS 2 were colocated with Tx antenna pointing towards RISs. Mode specific BLE packet waveforms are captured by Rx PlutoSDR which are
TABLE 1 | BLE system and radio parameters.

\begin{tabular}{lclc} 
BLE parameter & Value & Radio parameters & Value \\
\hline Samples/symbol & 8 & Tx Gain & $-25 \mathrm{~dB}$ \\
\hline Symbol Rate & $125 \mathrm{kbps}-2 \mathrm{Mbps}$ & Rx Gain & $10 \mathrm{~dB}$ \\
\hline Sample Rate & $8-16 \mathrm{MS} / \mathrm{s}$ & Gain Source & Manual
\end{tabular}

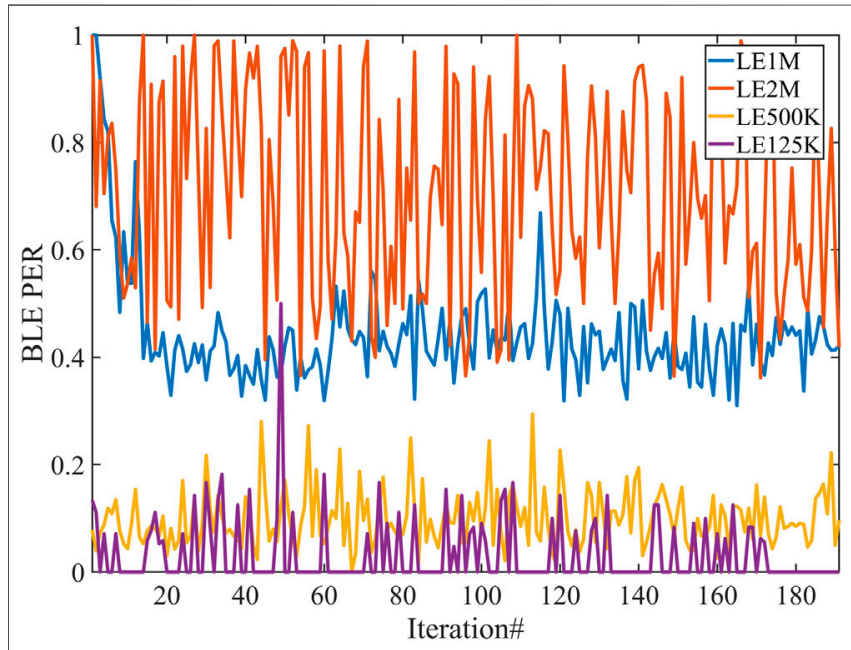

FIGURE 4 | PER measurement results of all the BLE PHY modes as a function of random RIS configuration. The PER measurements are performed with one RIS.

digital-downconverted to complex baseband samples for processing in the BLE baseband receiver.

\section{MEASUREMENT RESULTS}

The RIS manipulates impinging electromagnetic waves and increases signal power using quasi-passive beamforming at the receiver. Received signal power in RIS-assisted communication depends on transmitted signal power, transmit and receive 


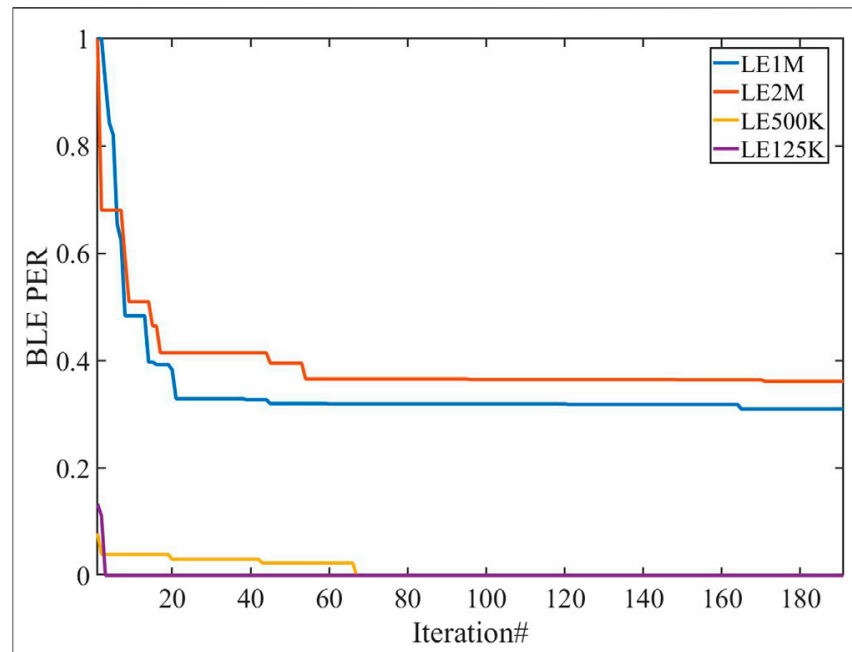

FIGURE 5 | Optimized PER results of all the BLE PHY modes as a function of optimized RIS configuration. The PER measurements are performed with one RIS.

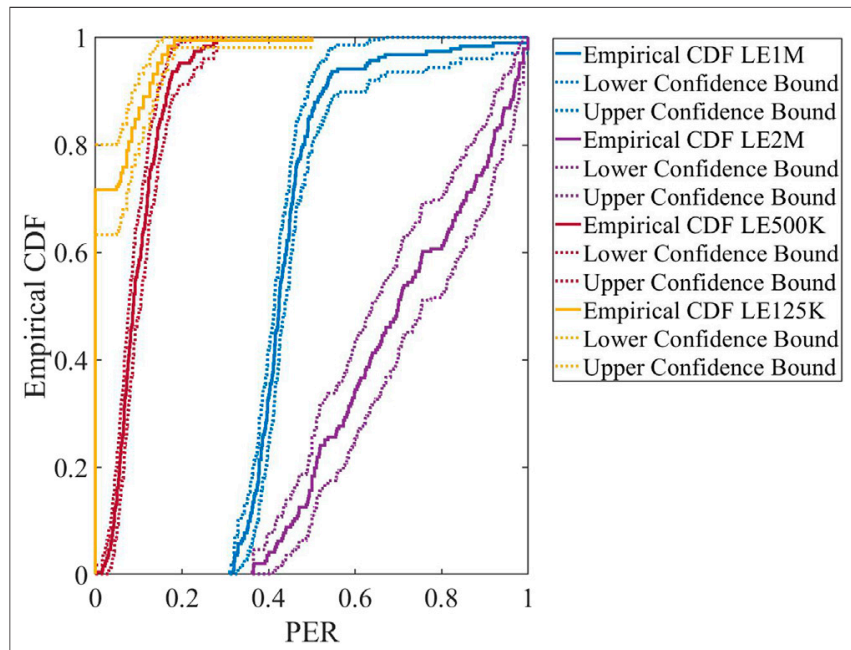

FIGURE 6 | Empirical CDF of PER measurements of all PHY modes of BLE using one RIS.

antenna gains and their polarization, gain and size of the unit cell, and frequency of operation. More importantly, it also depends on the method of RIS-element excitation. We performed PER for 190 random configurations using one RIS and 380 random configurations using two RISs. Random samples are generated for each measurement iteration. PER optimization is performed and the RIS values are considered which gives PER equal to or less than the current PER value. BLE system parameters and radio parameters are shown in Table. 1. PER performance of BLE PHY modes (see Figure 4) shows BLE LE2M PHY mode has worst PER followed by LE1M. LE1M and LE2M are uncoded PHY modes. Coded BLE PHY modes i.e., LE500K and LE125K show better PER than the uncoded LE1M and LE2M, respectively. The reason for better PER for coded BLE PHY modes is because of
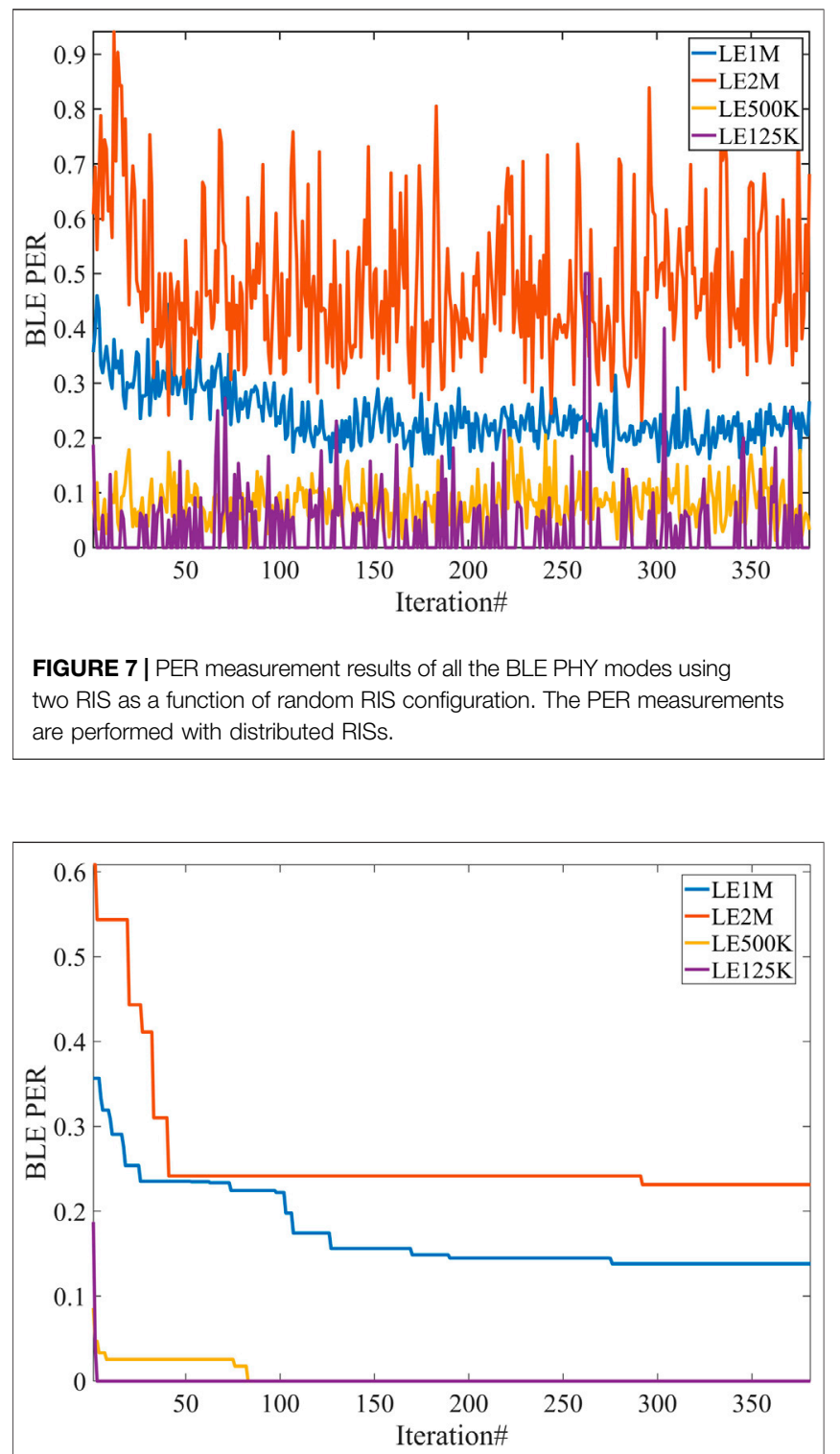

FIGURE 8 | PER measurement results of all the BLE PHY modes using two RIS as a function of optimized RIS configuration. The PER measurements are performed with distributed RISs.

the forward coding and low data rates. Similarly, among uncoded standard LE2M has higher data rate than the LE1M and because of higher data rates LE2M BLE link has higher PER. For initial RIS configuration PER for all BLE PHY modes is high as shown in Figure 5. After the RIS tunes its element properties, the PER for all BLE PHY modes improves. Figure $\mathbf{6}$ shows empirical CDF of PER of all BLE PHY modes with 99\% confidence interval. The coded BLE PHY mode outperforms uncoded BLE PHY modes with lowest PER for LE125K and highest PER for LE2M. Lastly, the PER for all the BLE PHY modes is optimized using reconfigurable intelligent surface.

Figure 7 shows PER of all the BLE PHY modes for $8 \times 38$ RIS configurations. Similarly, Figures 8, 9 show optimized PER and 

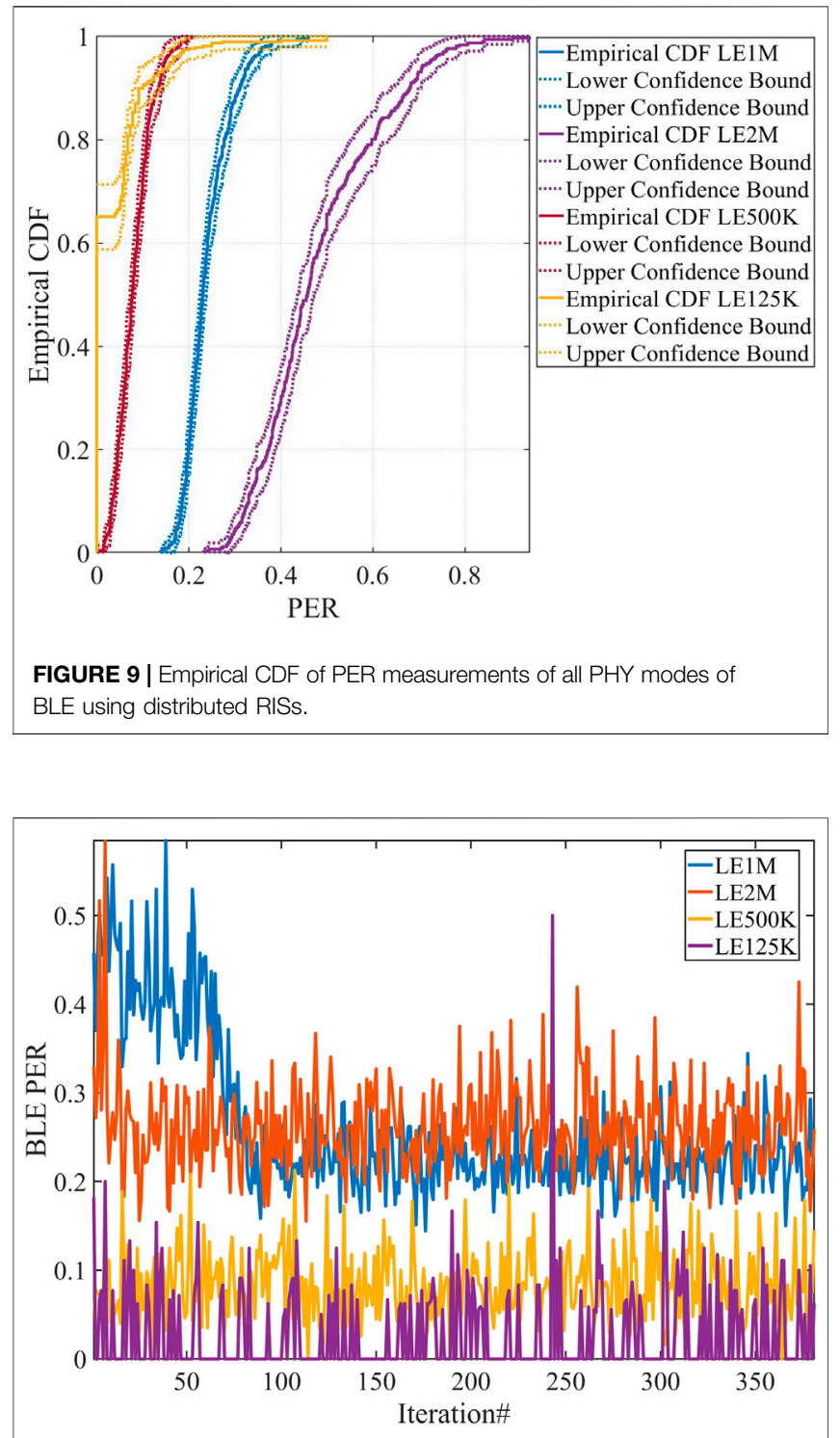

FIGURE 10|PER measurement results of all the BLE PHY modes using two RIS as a function of random RIS configuration. The PER measurements are performed with centralized RISs.

empirical CDF of PER of all the BLE PHY modes in distributed arrangement of RISs. PER measurements of all the BLE PHY modes in centralized RIS arrangement is shown in Figure 10. The average BLE PER for 2 Mbps LE2M mode is higher than rest of the BLE PHY modes both uncoded and coded. Coded LE500K and LE125K are superior in terms of PER performance. Similarly, Figures 8, 11 show optimized PER of all BLE PHY modes in presence of two RIS in distributed and centralized arrangement. Like our previous observations with one RIS, the LE2M PER using two RISs is higher than the PER of rest of PHY modes for both RISs arrangements. Optimized PER of LE1M is lower than the LE2M PER and is in accordance with the data-rate intuition. LE125K has the lowest PER of all BLE PHY modes (see Figures 9, 12). It can be noticed that the performance of centralized RISs is higher than than

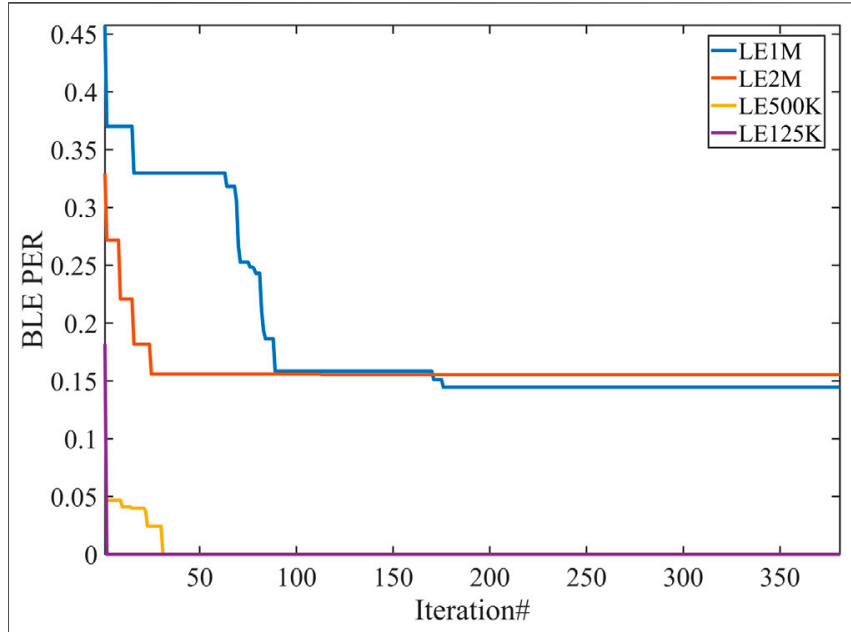

FIGURE 11 | PER measurement results of all the BLE PHY modes using two RIS as a function of optimized RIS configuration. The PER measurements done with centralized RISs.

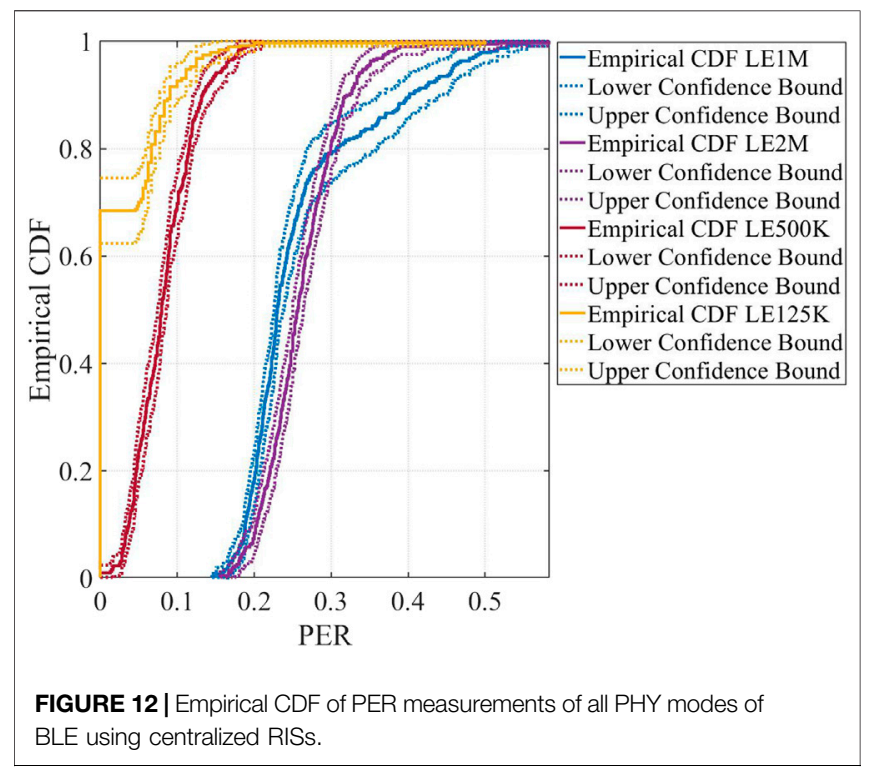

distributed RISs. The radiation pattern of the Tx Antenna intercepts only one RIS in the distributed scenario, while it intercepts both the RISs in the centralized scenario. Hence, the centralized scenario benefits from channel hardening.

From our measurements it is shown that the BLE link PER reliability increases when we increase the RIS size as shown in Figures 6, 9, 12, respectively. Additionally, the reliability of BLE link is higher for centralized arrangement than the distributed arrangement. Additionally, irrespective of RIS size the reliability of coded BLE PHY mode i.e. LE125K is highest than the rest of BLE PHY modes. Finally, it can be noticed that the uncoded BLE PHY modes LE2M reliability increases with the increase in RIS size. This suggests that the usage of RIS and increased size of RIS can sustain higher data rates. Figures 13-15 shows final best configuration using one RIS, and two RISs in distributed and centralized configurations respectively. 


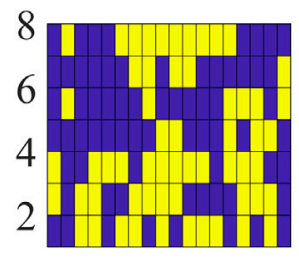

$5 \quad 10 \quad 15$

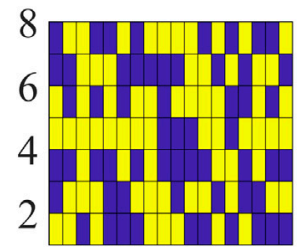

$5 \quad 10 \quad 15$

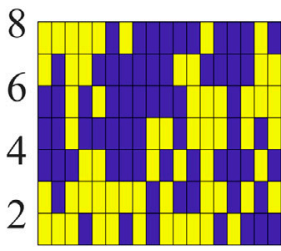

$\begin{array}{lll}5 & 10 & 15\end{array}$

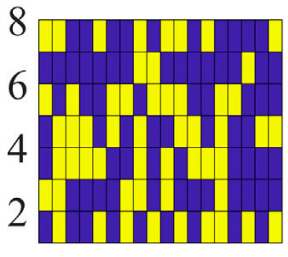

$\begin{array}{lll}5 & 10 & 15\end{array}$

FIGURE 13 | Optimized binary coding matrices representing final configurations of the RIS for LE1M, LE2M, LE500K, and LE125K.

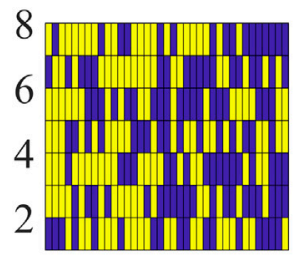

$10 \quad 20 \quad 30$

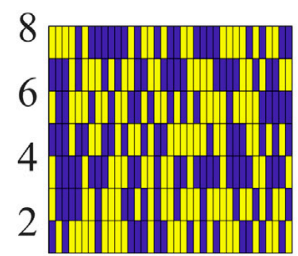

102030

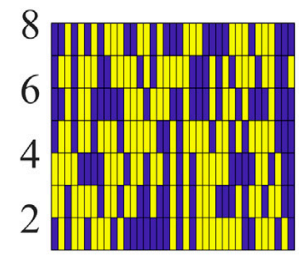

102030

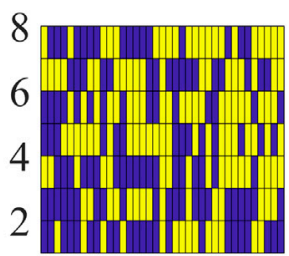

102030

FIGURE 14 | Optimized binary coding matrices representing final configurations of the two distributed RISs for LE1M, LE2M, LE500K, and LE125K.

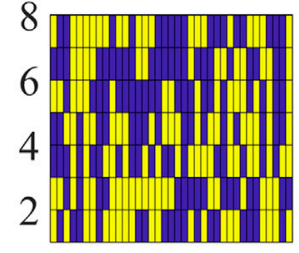

$10 \quad 20 \quad 30$

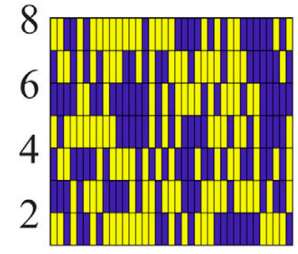

$1020 \quad 30$

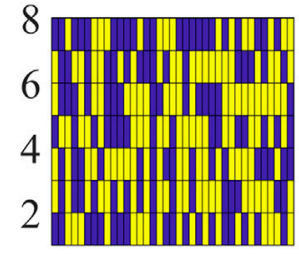

$10 \quad 2030$

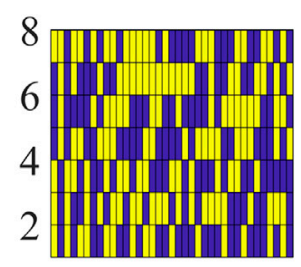

$10 \quad 2030$

FIGURE 15 | Optimized binary coding matrices representing final configurations of the two centralized RISs for LE1M, LE2M, LE500K, and LE125K.

These final configurations yield the optimized PER for all BLE PHY modes using one RIS and two RISs respectively.

\section{CONCLUSION}

In this work, we created RIS-assisted programmable radio environment, contrary to legacy radio channels, programmable radio channel creates a degree of freedom for PER optimization. We demonstrated RIS-assisted BLE communication link in metal enclosure. We checked the PER performance of four BLE PHY layer modes and optimized the BLE PER for all the BLE PHY modes. From measurement results we conclude that the RIS successfully optimized PER of all BLE PHY modes. Out of two RISs arrangements centralized arrangement is better than the distributed arrangement. One potential reason could be the path loss between transmitter and the RIS is higher in distributed arrangement than in the centralized arrangement where the Tx antenna pointing towards RISs illuminates RISs at same length with same power. Future aspect of the research is to perform PER optimization in presence of active RF interference signals.

\section{DATA AVAILABILITY STATEMENT}

The raw data supporting the conclusion of this article will be made available by the authors, without undue reservation.

\section{AUTHOR CONTRIBUTIONS}

ML performed measurements and covered mainly the SDR aspect of the research and did data analysis. J-BG created the measurement setup and the optimization code. GG directed reverberation and metal enclosure effects related aspects of the research. SG and GL arranged measurement equipment and presented some thoughts on the results.

\section{FUNDING}

This work was supported by the European Commission through the H2020 RISE-6G Project under Grant 101017011, and in part by the Royal Society under Grant INF\R2\192066. 


\section{REFERENCES}

Barazzetta, M., Micheli, D., Bastianelli, L., Diamanti, R., Totta, M., Obino, P., et al. (2017). "A Comparison Between Different Reception Diversity Schemes of a 4G-LTE Base Station in Reverberation Chamber: A Deployment in a Live Cellular Network," in IEEE Transactions on Electromagnetic Compatibility 59 (6), 2029-2037. doi:10.1109/TEMC.2017.2657122

Basar, E., Di Renzo, M., De Rosny, J., Debbah, M., Alouini, M.-S., and Zhang, R. (2019). Wireless Communications through Reconfigurable Intelligent Surfaces. IEEE access 7, 116753-116773. doi:10.1109/access.2019.2935192

Björnson, E., Özdogan, Ö., and Larsson, E. G. (2019). Intelligent Reflecting Surface versus Decode-And-Forward: How Large Surfaces Are Needed to Beat Relaying? IEEE Wireless Commun. Lett. 9, 244-248. doi:10.1109/ LWC.2019.2950624

Boulogeorgos, A.-A. A., and Alexiou, A. (2020). Performance Analysis of Reconfigurable Intelligent Surface-Assisted Wireless Systems and Comparison with Relaying. IEEE Access 8, 94463-94483. doi:10.1109/ access.2020.2995435

Calvanese Strinati, E., Alexandropoulos, G. C., Sciancalepore, V., Renzo, M. D., Wymeersch, H., Phan-huy, D.-T., et al. (2021). "Wireless Environment as a Service Enabled by Reconfigurable Intelligent Surfaces: The RISE-6G Perspective," in Proc. Of EUCNC 6G Summit (Porto, Portugal: IEEE). doi:10.1109/eucnc/6gsummit51104.2021.9482474

Cui, M., Zhang, G., and Zhang, R. (2019). Secure Wireless Communication via Intelligent Reflecting Surface. IEEE Wireless Commun. Lett. 8, 1410-1414. doi:10.1109/lwc.2019.2919685

Dai, L., Wang, B., Wang, M., Yang, X., Tan, J., Bi, S., et al. (2020). Reconfigurable Intelligent Surface-Based Wireless Communications: Antenna Design, Prototyping, and Experimental Results. IEEE Access 8, 45913-45923. doi:10.1109/access.2020.2977772

Gagliardi, L., Micheli, D., Gradoni, G., Moglie, F., and Primiani , V. M. (2015). "Coupling Between Multipath Environments Through a Large Aperture," in IEEE Antennas and Wireless Propagation Letters 14, 1463-1466. doi:10.1109/ LAWP.2015.2411621

Guan, X., Wu, Q., and Zhang, R. (2020). Joint Power Control and Passive Beamforming in Irs-Assisted Spectrum Sharing. IEEE Commun. Lett. 24, 1553-1557. doi:10.1109/lcomm.2020.2979709

Hou, T., Liu, Y., Song, Z., Sun, X., Chen, Y., and Hanzo, L. (2019). Mimo Assisted Networks Relying on Large Intelligent Surfaces: A Stochastic Geometry Model. arXiv preprint arXiv:1910.00959, 1.

Hu, J., Zhang, H., Di, B., Li, L., Bian, K., Song, L., et al. (2020). Reconfigurable Intelligent Surface Based Rf Sensing: Design, Optimization, and Implementation. IEEE J. Select. Areas Commun. 38, 2700-2716. doi:10.1109/ jsac.2020.3007041

Huang, C., Zappone, A., Alexandropoulos, G. C., Debbah, M., and Yuen, C. (2019). Reconfigurable Intelligent Surfaces for Energy Efficiency in Wireless Communication. IEEE Trans. Wireless Commun. 18, 4157-4170. doi:10.1109/twc.2019.2922609

Kaina, N., Dupré, M., Lerosey, G., and Fink, M. (2014b). Shaping Complex Microwave fields in Reverberating media with Binary Tunable Metasurfaces. Sci. Rep. 4, 6693-6698. doi:10.1038/srep06693

Kaina, N., Dupré, M., Fink, M., and Lerosey, G. (2014a). Hybridized Resonances to Design Tunable Binary Phase Metasurface Unit Cells. Opt. Express 22, 18881-18888. doi:10.1364/oe.22.018881

Li, S., Duo, B., Yuan, X., Liang, Y.-C., and Di Renzo, M. (2020). Reconfigurable Intelligent Surface Assisted Uav Communication: Joint Trajectory Design and Passive Beamforming. IEEE Wireless Commun. Lett. 9, 716-720. doi:10.1109/ lwc.2020.2966705

Litwin, L. (2001). Matched Filtering and Timing Recovery in Digital Receivers. $R F$ Des. 24, 32-49.

Lodro, M., Gradoni, G., Vukovic, A., Thomas, D., and Greedy, S. (2021). Per Measurement of Ble in Rf Interference and Harsh Electromagnetic Environment. arXiv e-prints, arXiv-2105.
Lodro, M., Smart, C., Gradoni, G., Vukovic, A., Thomas, D., and Greedy, S. (2020) Near-field ber and evm measurement at $5.8 \mathrm{ghz}$ in mode-stirred metal enclosure. Appl. Comput. Electromagnetics Soc. J. 35, 228919015. doi:10.47037/2020.aces.j.350915

Micheli, D., Barazzetta, M., Bastianelli, L., Diamanti, R., Carlini, C., Colombo, M., et al. (2021). "MIMO $4 \times 4$ vs. MIMO $2 \times 2$ Performance Assessment of a Real Life LTE Base Station in a Reverberation Chamber. Int. J. Electron. Commun. 129,153500

Micheli, D., Barazzetta, M., Carlini, C., Diamanti, R., Primiani , V. M., and Moglie, F. (2016). "Testing of the Carrier Aggregation Mode for a Live LTE Base Station in Reverberation Chamber," in IEEE Transactions on Vehicular Technology 66 (4), 3024-3033. doi:10.1109/TVT.2016.2587662

Montoliu, R., Sansano, E., Gascó, A., Belmonte, O., and Caballer, A. (2020). Indoor Positioning for Monitoring Older Adults at home: Wi-Fi and Ble Technologies in Real Scenarios. Electronics 9, 728. doi:10.3390/electronics9050728

Primiani, V. M., Barazzetta, M., Bastianelli, L., Micheli, D., Moglie, E., Diamanti, R., et al. (2020). "Reverberation Chambers for Testing Wireless Devices and Systems," in IEEE Electromagnetic Compatibility Magazine 9 (2), 45-55. doi:10.1109/MEMC.2020.9133241

Spachos, P., and Plataniotis, K. (2020). Ble Beacons in the Smart City: Applications, Challenges, and Research Opportunities. IEEE Internet Things M. 3, 14-18. doi:10.1109/iotm.0001.1900073

Tan, X., Sun, Z., Koutsonikolas, D., and Jornet, J. M. (2018). "Enabling Indoor mobile Millimeter-Wave Networks Based on Smart Reflect-Arrays," in IEEE INFOCOM 2018-IEEE Conference on Computer Communications (IEEE), 270-278. doi:10.1109/infocom.2018.8485924

Tang, W., Chen, M. Z., Chen, X., Dai, J. Y., Han, Y., Di Renzo, M., et al. (2020a). Wireless Communications with Reconfigurable Intelligent Surface: Path Loss Modeling and Experimental Measurement. IEEE Trans. Wireless Commun. 99, 1. doi:10.1109/TWC.2020.3024887

Tang, W., Dai, J. Y., Chen, M. Z., Wong, K.-K., Li, X., Zhao, X., et al. (2020b). Mimo Transmission through Reconfigurable Intelligent Surface: System Design, Analysis, and Implementation. IEEE J. Select. Areas Commun. 38, 2683-2699. doi:10.1109/jsac.2020.3007055

Wu, Q., and Zhang, R. (2019). Towards Smart and Reconfigurable Environment: Intelligent Reflecting Surface Aided Wireless Network. IEEE Commun. Mag. 58, 106-112. doi:10.1109/MCOM.001.1900107

$\mathrm{Xu}, \mathrm{S}$., Liu, J., and Cao, Y. (2021). "Intelligent Reflecting Surface Empowered Physical Layer Security: Signal Cancellation or Jamming?," in IEEE Internet of Things Journal (IEEE), 1. doi:10.1109/jiot.2021.3079325

Yuan, X., Zhang, Y.-J. A., Shi, Y., Yan, W., and Liu, H. (2021). Reconfigurableintelligent-surface Empowered Wireless Communications: Challenges and Opportunities. IEEE Wireless Commun. 28, 136-143. doi:10.1109/mwc.001.2000256

Conflict of Interest: Authors J-BG and GL were employed by the company Greenerwave.

The remaining author declares that the research was conducted in the absence of any commercial or financial relationships that could be construed as a potential conflict of interest.

Publisher's Note: All claims expressed in this article are solely those of the authors and do not necessarily represent those of their affiliated organizations, or those of the publisher, the editors and the reviewers. Any product that may be evaluated in this article, or claim that may be made by its manufacturer, is not guaranteed or endorsed by the publisher.

Copyright $\odot 2021$ Lodro, Gradoni, Gros, Greedy and Lerosey. This is an open-access article distributed under the terms of the Creative Commons Attribution License (CC $B Y)$. The use, distribution or reproduction in other forums is permitted, provided the original author(s) and the copyright owner(s) are credited and that the original publication in this journal is cited, in accordance with accepted academic practice. No use, distribution or reproduction is permitted which does not comply with these terms. 